\title{
151 years Berliner Klinische Wochenschrift and the 20th anniversary of the Journal of Molecular Medicine
}

\author{
Detlev Ganten ${ }^{1} \cdot$ Gregg Semenza ${ }^{2} \cdot$ Thomas Sommer $^{3}$
}

Received: 13 July 2015 / Accepted: 15 July 2015 / Published online: 11 August 2015

(C) Springer-Verlag Berlin Heidelberg 2015

We are very proud that the Journal of Molecular Medicine ( $J$ $\mathrm{Mol} \mathrm{Med}$ ) celebrates its twentieth anniversary in 2015. The story of the remarkable growth and impact of the $J \mathrm{Mol}$ Med begins with the Berliner Klinische Wochenschrift, which was introduced in 1864 at the time natural sciences entered medical research and when the famous "Berliner Schule" of medicine began. As one of the oldest and most prestigious European journals, in which great scientists such as Paul Ehrlich, Robert Koch, and Otto Warburg published their seminal, epoch-making discoveries, Berliner Klinische Wochenschrift is the prestigious predecessor of $J \mathrm{Mol} \mathrm{Med}$. The name of the journal was shortened to Klinische Wochenschrift (Clinical Weekly) in 1922, and subsequently changed to The Clinical Investigator with exclusively English language contributions in 1992. In 1995, the editorial office moved to the Max Delbrück Center for Molecular Medicine (MDC) in BerlinBuch, Germany, and the journal was renamed the Journal of Molecular Medicine (J Mol Med) [1].

Since 1995, J Mol Med has been successful in bringing together basic science and clinical medicine with a clear focus on molecular biology, gene technology, genetics, genomics, proteomics, bioinformatics, and the translation of basic biological and medical research into clinical practice. At this time, 20 years ago, $J \mathrm{Mol} \mathrm{Med}$ was among the first journals to emphasize the

Detlev Ganten

jmmeic@mdc-berlin.de

1 Charité-Universitätsmedizin, Charitéplatz 1, 10117 Berlin, Germany

2 Institute for Cell Engineering, The Johns Hopkins University School of Medicine, 733 North Broadway, Baltimore, MD 21205, USA

3 Max Delbrück Center for Molecular Medicine in the Helmholtz Association, Robert-Rössle Straße 10, 13125 Berlin, Germany focus on molecular medicine and to foster the translational approach, with the goal to accelerate the development of new diagnostic tools and therapies from the bench to the bedside and move the field of molecular medicine forward. Especially in recent years, numerous other journals were launched that commit to the topic of molecular medicine and translation. This reflects the growing interest and demand for fast translation of basic findings into clinical relevance and eventually to the better health of society. For us as Editors, Associate Editors, and Editorial Board, this also means that we strengthen our commitment to compete for the best submissions in order to maintain and improve the standards of $J \mathrm{Mol} \mathrm{Med}$.

Building a strong scientific community of readers, authors, and supporters for the journal is one way to maintain the high quality and flow of excellent submissions. $\mathrm{J} \mathrm{Mol} \mathrm{Med} \mathrm{is} \mathrm{not}$ the journal of one scientific society or institution, but it has the support of a strong international community of scientists and a strong relationship with a unique academic network, the "M8 Alliance of Academic Health Centers, Universities and National Academies." The M8 Alliance includes National and Medical Academies in more than 80 countries and Academic Health Centers in the USA, the UK, Canada, France, Germany, Russia, Japan, Australia, Singapore, and Brazil - a unique network of excellence around the world, of outstanding, topranking institutions and researchers. This collaboration of prestigious international medical universities and academies is committed to improving global health through scientific, evidence-based medicine and to promoting translation from bench, to bedside, to better health in populations. It also stimulates international exchange at the annual World Health Summit (WHS) held in Berlin and regular WHS Regional Meetings around the world (www.worldhealthsummit.org).

Leading scientists from these institutions have joined our Editorial Board already, and we aim to foster further communication and collaboration with these institutions in the future. 
Moreover, $J$ Mol Med aims to strengthen its relationship with individual high-class institutes such as the newly established Berlin Institute of Health (BIH, https://www.bihealth.org/en/). This is a joint venture of the Max Delbrück Center for Molecular Medicine (MDC) Berlin-Buch and the CharitéUniversitätsmedizin Berlin. New top-level international scientists with a systems medicine and translational research orientation are being recruited by the $\mathrm{BIH}$, with a view to bridge the gap between basic research and clinical application.

Now, with the rapid development of high throughput technologies, the future certainly lies in detailed molecular analysis but especially also in systems-oriented approaches. The systems approach considers all major diseases as systemic diseases originating from a combination of malfunctioning pathways or organs, and not from a single isolated patho-mechanism. Certain molecular pathways are often involved in many different diseases, for instance, atherosclerosis, inflammatory bowel disease, neurodegeneration, and cancer all involve an inflammatory component. A better understanding of the molecular causes of inflammatory reactions will foster the development of new therapies and cures. This applies to many more basic mechanisms of molecular medicine. The systems approach integrates -omics data with mathematical and bioinformatical modeling to make comprehensive molecular characterization of diseases possible. This will hopefully open completely new avenues for the development of tailored diagnostics and therapies as well as personalized precision medicine for major common diseases. $\mathrm{J} \mathrm{Mol} \mathrm{Med} \mathrm{wishes} \mathrm{to} \mathrm{broaden}$ its focus on systems medicine and therefore welcomes submissions from this interdisciplinary area.

Now entering its third decade, $J$ Mol Med is still among the most read journals at the forefront of molecular medicine. This is due to combined efforts of a strong editorial team and an Editorial Board with a broad range of expertise in all areas relevant to molecular medicine, a dedicated editorial office in Berlin, as well as a strong liaison with the Springer Publishing and last but not least, many highly professional colleagues who act as referees for the journal. Their entirely voluntary work not only maintains the high scientific standards of the journal but also, through their comments and reviews of the manuscripts, provides authors with the opportunity to improve the quality of their submissions.

To cope with the workload and to guarantee a professional handling of the diverse submissions, we have recently restructured our Editorial Board. The team of Editors in Chief, supported by the team of Managing Editors in Berlin, work with 16 Associate Editors to handle submissions from the different areas of molecular medicine. In addition, we recruited several Review Editors who give guidance on the most interesting topics in their fields and handle review articles.

Walter Rosenthal, who was Co-Editor in Chief since 2009 and was active for the Journal as Associate Editor many years before, was recently appointed President of the University of Jena. We thank him for his continuous input and support of the editorial work. We are very pleased that the interim Scientific Director of the Max Delbrück Center for Molecular Medicine in the Helmholtz Association, Thomas Sommer, followed Walter Rosenthal in his capacity as Chief Editor. Thomas Sommer and his laboratory made significant contributions to the role of the ubiquitin proteasome pathways in proteolysis and at the endoplasmic reticulum. The focus of his current research is directed at understanding the interplay between ubiquitin ligases and molecular chaperones in protein quality control pathways, which has implications for many diseases.

The success of the Journal is reflected by its respectable impact factor of 5.107 (ISI 2014) and its high visibility and broad readership (more than 10,000 institutions worldwide have full text access). Apart from these factors and its proud and long tradition, there are several good reasons to publish in $J \mathrm{Mol}$ Med. One is quality - we are convinced that the peer-review process is critical to the quality of a journal and thus we will stick to this procedure. In an age of rapid electronic publication, it is the peer-review process and the work of the expert referees that differentiates scientific journals from e-print archives.

In order to expand the scope of $J \mathrm{Mol} \mathrm{Med}$, we introduced a new review series entitled "Molecules in Medicine" in 2014. The series is aimed to provide a comprehensive overview of an individual molecule (or a well-defined "family" of molecules), its molecular function, and implications for human disease. It also provides an excellent chance to give a crisp and clear overview of the historic background of a class of molecules, for the benefit of those who did not witness the times of discovery. We anticipate that the "Molecules in Medicine" review series will provide a highly cited and comprehensive knowledge base that will serve many readers and authors with comprehensive and up-to-date information on specific molecules and their functional relevance for human disease.

We very much hope that our authors and readers will continue to consider $J \mathrm{Mol} \mathrm{Med}$ for publication of their important scientific work.

\section{Reference}

1. Ganten D, Vogt P (2000) JMM and medical science, past and present. J Mol Med 78:397-399 